\title{
An animal model of fetal alcohol spectrum disorder: Trace conditioning as a window to inform memory deficits and intervention tactics
}

\author{
Pamela S. Hunt \\ College of William and Mary \\ Robert C. Barnet \\ College of William and Mary
}

Follow this and additional works at: https://scholarworks.wm.edu/aspubs

\section{Recommended Citation}

Hunt, P. S., \& Barnet, R. C. (2015). An animal model of fetal alcohol spectrum disorder: Trace conditioning as a window to inform memory deficits and intervention tactics. Physiology \& behavior, 148, 36-44.

This Article is brought to you for free and open access by the Arts and Sciences at W\&M ScholarWorks. It has been accepted for inclusion in Arts \& Sciences Articles by an authorized administrator of W\&M ScholarWorks. For more information, please contact scholarworks@wm.edu. 


\title{
An animal model of fetal alcohol spectrum disorder: Trace conditioning as a window to inform memory deficits and intervention tactics
}

\author{
Pamela S. Hunt *, Robert C. Barnet \\ Department of Psychology, College of William \&'Mary, USA
}

\section{H I G H L I G H T S}

- A rodent model of FASD exhibits impaired trace conditioning

- Environmental, nutritional and pharmacological interventions were examined

- Associative learning procedures are useful for probing cognitive impairments in FASD

- Trace conditioning deficits can be used to screen treatments for FASD

\section{A R T I C L E I N F O}

\section{Article history:}

Received 12 September 2014

Received in revised form 21 November 2014

Accepted 23 November 2014

Available online 2 December 2014

\section{Keywords:}

Fetal alcohol

Trace conditioning

Intervention

Choline

Physostigmine

Serial compound

\begin{abstract}
A B S T R A C T
Animal models of Fetal Alcohol Spectrum Disorders (FASD) afford the unique capacity to precisely control timing of alcohol exposure and alcohol exposure amounts in the developing animal. These models have powerfully informed neurophysiological alterations associated with fetal and perinatal alcohol. In two experiments presented here we expand use of the Pavlovian Trace Conditioning procedure to examine cognitive deficits and intervention strategies in a rat model of FASD. Rat pups were exposed to $5 \mathrm{~g} / \mathrm{kg} /$ day ethanol on postnatal days (PD) 4-9, simulating alcohol exposure in the third trimester in humans. During early adolescence, approximately PD 30, the rats were trained in the trace conditioning task in which a light conditioned stimulus (CS) and shock unconditioned stimulus (US) were paired but separated by a 10-s stimulus free trace interval. Learning was assessed in freezing behavior during shock-free tests. Experiment 1 revealed that neonatal ethanol exposure significantly impaired hippocampus-dependent trace conditioning relative to controls. In Experiment 2 a serial compound conditioning procedure known as 'gap filling' completely reversed the ethanol-induced deficit in trace conditioning. We also discuss prior data regarding the beneficial effects of supplemental choline and novel preliminary data regarding the pharmacological cognitive enhancer physostigmine, both of which mitigate the alcoholinduced cognitive deficit otherwise seen in trace conditioning controls. We suggest trace conditioning as a useful tool for characterizing some of the core cognitive deficits seen in FASD, and as a model for developing effective environmental as well as nutritional and pharmacological interventions.
\end{abstract}

(c) 2014 Elsevier Inc. All rights reserved.

\section{Introduction}

Exposure to high doses of ethanol during gestation can lead to Fetal Alcohol Syndrome (FAS), defined by three core diagnostic criteria: (a) preand post-natal growth retardation, (b) craniofacial dysmorphologies, and (c) central nervous system dysfunction [40]. A large number of individuals prenatally exposed to alcohol do not meet all three criteria for a FAS diagnosis, and have been labeled as having partial FAS, alcoholrelated birth defects (ARBD), and/or alcohol-related neurodevelopmental disorders (ARND) [83]. Collectively these conditions now fall under the

\footnotetext{
* Corresponding author at: Department of Psychology, College of William \& Mary, P.O Box 8795, Williamsburg, VA 23187-8795, USA. Tel.: +1 757221 3894; fax: +1 757221 3896.

E-mail address: pshunt@wm.edu (P.S. Hunt).
}

umbrella term of Fetal Alcohol Spectrum Disorder (FASD; [31]). It has been estimated that the prevalence of FASD conditions could be as high as 10 in 1000 live births $[56,80]$.

Alcohol causes severe central nervous system dysfunction, especially when exposure occurs during early brain development. Alcohol itself has been identified as a teratogen, affecting the developing individual in a variety of ways. While many brain regions are negatively affected by alcohol, some of the regions which are particularly vulnerable to ethanol toxicity are the cerebellum, hippocampus, striatum and frontal cortex $[13,76,98]$. These regions are known to be involved in attention, learning, memory, executive cognitive function, and social behaviors, which are some of the primary functional impairments noted in individuals characterized along the fetal alcohol spectrum [44-46,53,67].

In humans, individuals exposed to even modest amounts of alcohol during gestation can exhibit a wide range of dysfunctions within several 
neuropsychological domains $[14,51,83,84]$ and some of these effects appear to be permanent, persisting into adulthood [13,47,98]. Some areas of cognitive functioning are more affected than others [50,54]; indeed some processes may even be spared from ethanol toxicity while others show significant impairment. Mattson and Riley [52], for example, have shown that children with prenatal exposure to alcohol exhibit deficits on explicit, but not implicit, memory tasks. Relatively selective alcohol effects on measures of declarative, but not procedural, memory have also been reported [69,79]. Uecker and Nadel [96] noted impairments in spatial, but not nonspatial, memory in children diagnosed with FAS (see also [27]). Furthermore, deficits expressed by ethanol-exposed individuals vary with task demands and tend to be exacerbated as task complexity increases [45]. Given the variability of ethanol's effects in humans, it has been important to develop well controlled models of ethanol's effects on cognition and neurophysiology.

Animal research has contributed vitally to our understanding of the mechanisms of toxicity as well as the behavioral consequences of fetal alcohol exposure in well-controlled experiments. Much of this research has involved characterizing alcohol's effects on the hippocampus. It is known that ethanol causes structural damage to this region, including decreased hippocampal volume, reductions in cell number and density, altered synaptic morphology, and altered intracellular signaling mechanisms $[11,20,59,68]$. Hippocampal plasticity in the form of long-term potentiation, a putative mechanism for memory formation, is also reduced following early alcohol exposure $[72,86]$.

Regional differences in brain vulnerability to alcohol insult depend on the timing of ethanol exposure relative to critical periods of brain development (e.g. [24,94]). Administration of ethanol during the early postnatal period is used to model the consequences of third-trimester ethanol exposure in humans. The third trimester in humans is a period described by Dobbing and Sands [16] as the "brain growth spurt." Many cognitive processes are subserved by brain structures that are known to exhibit considerable maturation during this time, including the hippocampus. In rodents, a roughly equivalent period of brain growth and maturation occurs postnatally, during the first two weeks following birth. For many animal researchers using rodents to model ethanolinduced cognitive impairments, ethanol is administered during this postnatal brain growth period, typically on Postnatal Days (PD) 4-9.

This method of alcohol exposure has been used successfully by many laboratories to obtain neural and behavioral changes that model those observed in humans. It is important to emphasize that the model is used to obtain the neural and behavioral outcomes of neonatal ethanol exposure, in our case an impairment in cognitive function (i.e., learning), not the precise manner of alcohol exposure in humans. While human fetuses are more likely to be exposed to alcohol during the early phases of gestation, or throughout all of gestation, there are several studies that corroborate the impact of alcohol during the third trimester as most critical to causing cognitive impairments [6,47]. Rodent research has verified the particular vulnerability of the hippocampus to postnatal ethanol exposure as well $[49,94]$.

Many behavioral studies have brought focus to the impact of early alcohol on the function of memory systems involving the hippocampus, and suggest a variety of memory processes are permanently affected by early alcohol exposure. There are numerous reports of learning and memory deficits in animal subjects exposed to ethanol during the perinatal (pre- and/or postnatal) period. In many respects, the results obtained with animal models parallel the findings from the clinical literature on human FASD. For example, animals exposed to ethanol during the perinatal period exhibit altered spatial memory performance, while performance in nonspatial, control versions of these tasks is generally unaffected (e.g. $[21,24,65,88,89])$. Contextual fear conditioning, another hippocampus dependent task, can be especially compromised by neonatal ethanol exposure, even though learning about a concomitant conditioned stimulus (CS) is unaffected $[23,63,64]$.

Another consistent finding is an ethanol impairment in short-term or working memory processes, which has been tested in several paradigms $[21,25,65,66]$. For the past 15 years, our laboratory has been working to better characterize ethanol-induced deficits in various aspects of short-term memory. We have examined short-term recognition memory through the measurement of habituation of the heart rate orienting response to a novel olfactory stimulus $[33,35,60]$ and shortterm/working memory functions through the use of a trace fear conditioning paradigm $[34,82,97]$. In the present paper, we briefly review some of our previous results and extend use of the Pavlovian trace conditioning procedure by presenting novel findings that further characterize the effects of neonatal alcohol and suggest mitigating interventions.

There are many variants of the Pavlovian conditioning paradigm. In what is called the delay procedure, the offset of the conditioned stimulus (CS) is coincident with presentation of the unconditioned stimulus (US). The amygdala is the brain structure most often associated with delay fear conditioning [48], in which an aversive stimulus such as footshock serves as the US. The trace procedure is similar, in that a CS and US are explicitly paired; however, the offset of the CS and onset of the US are separated by a stimulus-free period known as the trace interval. Conditioned responding typically declines as the trace interval is lengthened $[9,34,62]$. Interference with normal hippocampal function has been shown to affect acquisition of trace conditioning [9,39,42,62, 73 ]. The late ontogenetic emergence of trace, relative to delay, conditioning has also been interpreted as reflecting hippocampal immaturity $[1,38,61]$. Clark et al. [10] argue that trace conditioning involves a hippocampal declarative memory system, which supports the conjecture that trace conditioning can be used in animal research to explore declarative memory impairments resulting from perinatal ethanol exposure [17].

In the present research we first demonstrate that neonatal ethanol produces a trace fear conditioning deficit in rats, relative to controls. We then focus on three methods that can improve this type of learning despite ethanol exposure. Specifically, in Experiment 1 the effects of neonatal ethanol on trace and delay fear conditioning was assessed. Experiment 2 examined the possibility that an environmental intervention could attenuate the ethanol-induced trace conditioning impairment. Finally, we discuss some research involving targeted nutritional (choline) and pharmacological (physostigmine) treatments that also mitigate the selective ethanol-induced impairment in trace conditioning.

\section{General method}

\subsection{Subjects}

Sprague-Dawley derived rats served as subjects. Animals were born and reared in the vivarium at the College of William \& Mary. Animals were descendants of breeders originally obtained from Charles River Laboratories (Wilmington, MA). Breeder pairs were maintained in $50.8 \times 40.6 \times 21.6 \mathrm{~cm}$ clear polycarbonate cages with wire lids and pine chip bedding. The animal room was maintained on a $14: 10 \mathrm{~h}$ light cycle with lights on at $0600 \mathrm{~h}$. The room was temperature and humidity controlled. Animals had ad lib access to high-protein rat chow (Lab Diet Formula 5008, PMI Nutrition International, Brentwood, MO) and water at all times. The day of birth was designated as postnatal day (PD) 0 and litters were culled to 8-10 pups on PD 2. All procedures were approved by the Institutional Animal Care and Use Committee at the College of William \& Mary.

Pups began ethanol exposure treatment on PD 4, with half of the animals from each litter assigned to receive ethanol and the other half assigned to receive sham intubations. In some experiments we included an Unhandled control group, and those pups were selected from different litters that remained untreated until the day of behavioral training. Pups were weaned on PD 21 and were group-housed with siblings throughout the experiments. No more than two pups (one male and one female) from each litter were assigned to a particular experimental group, and sex was always included in the analyses. Thus, except for sex, the litter served as the unit for data analysis [30]. 


\subsection{Apparatus}

\subsubsection{Training chambers}

Conditioning occurred in two identical modified Skinner boxes housed in sound-attenuating shells. A 4-W red bulb was mounted on an inner wall of the shell to provide low-level illumination. The two shorter walls of the conditioning chamber were stainless steel and the front and back walls and ceiling were made from clear Plexiglas. The floor of the chamber was constructed of stainless steel bars $0.5 \mathrm{~mm}$ in diameter and spaced $0.5 \mathrm{~mm}$ apart. The grid floor was connected to a custom built shock generator. The US was a 1-s presentation of a $0.5 \mathrm{~mA}$ constant current shock. The CS was a 10-s flashing light $(.25 \mathrm{~s}$ $\mathrm{ON} / .25 \mathrm{~s}$ OFF), produced by a $25-\mathrm{W}$ white incandescent bulb. The light was located outside the conditioning chamber centered in the middle of the back wall.

\subsubsection{Testing chambers}

Testing occurred in a novel context located in another room of the laboratory. A novel context was used for testing to eliminate any contaminating contribution of context fear (freezing) acquired during light-shock training on fear (freezing) elicited by the CS during test. The test chamber was constructed of clear Plexiglas and was open at the top and bottom. The chamber was 29.0 (high) $\times 21.5 \times 21.5 \mathrm{~cm}$ and rested on a piece of Plexiglas covered with brown paper. The chamber was housed in a sound-attenuating shell with a 7-W white bulb mounted on an inner wall to provide constant low-level illumination. The flashing light during testing was identical to that used for training and was positioned outside the testing chamber centered in the middle of the back wall.

PC computers were used to interface Coulbourn Instruments (Allentown, PA) software and hardware and control stimulus presentations during training and testing. Test sessions were videotaped using a Sony camera (model CCD-TRV67).

\subsection{Procedure}

\subsubsection{Ethanol administration}

Details of our ethanol administration procedures can be found in our previous papers [34,97]. Briefly, $5 \mathrm{~g} / \mathrm{kg} / \mathrm{day}$ ethanol was administered via intragastric intubation to neonatal rats, beginning on PD 4 and continuing through PD 9, using procedures adapted from Pierce et al. [71]. During each exposure session, all pups were removed from the home cage and placed in a heated holding cage with pine chip bedding. Each animal was then administered ethanol or given a sham intubation using PE-10 tubing attached to $1 \mathrm{cc}$ syringes, and then all pups were returned to the home cage. For Sham-intubated controls, no fluid was delivered (e.g. [24,55]). For Ethanol-treated subjects, the daily dose of ethanol was divided into two administrations of $2.5 \mathrm{~g} / \mathrm{kg}$ each, separated by $2 \mathrm{~h}$. The ethanol solution was $11.9 \% \mathrm{v} / \mathrm{v}$ dissolved in Similac ${ }^{\mathrm{TM}}$. A third intubation of the Similac ${ }^{\mathrm{TM}}$ vehicle was given $2 \mathrm{~h}$ later. Thus, the litter was removed from the home cage three times daily, for approximately 20 min each.

\subsubsection{Behavioral training}

Conditioning sessions involved a series of five CS-US pairings during a 30 -min session. The CS was a 10 -s flashing $25-\mathrm{W}$ white bulb, and the US was a 1-s $0.5 \mathrm{~mA}$ shock delivered through the grid floor of the experimental chamber. Inter-trial intervals (US offset-to-CS onset on the next trial) varied from 200 to 300 s. In Delay conditioning the US was delivered at offset of the CS. In Trace conditioning a trace interval of $10 \mathrm{~s}$ separated CS offset from US onset (cf. [1]).

\subsubsection{Testing}

All animals were tested in a novel context $24 \mathrm{~h}$ after training. Testing involved an extinction procedure, in which the target CS was repeatedly presented without the US. Performance during the test was measured as a percent freezing score. Freezing, a species-typical defensive response, is defined as the absence of observable movement except that required for respiration [18]. Test sessions were videotaped and a time-sampling method was used to score freezing. The animal was briefly observed at 2-s intervals during presentation of the CS and the number of intervals scored as freezing over the total number of observation periods was converted into a percentage. The percentage of freezing observed during a 10 -s pre-stimulus period was also recorded and was subtracted from the percentage of freezing observed during the 10 -s CS, producing a percent change score.

\subsubsection{Age of training and testing}

Behavioral training and testing was initiated in adolescence, typically at 30 days of age ( \pm 1 day), and there is a particular reason for using this age group. In our experience, adolescent animals show a particular propensity for fear conditioning, exhibiting rather high levels of CSelicited freezing following modest training. More importantly, adolescents have been shown to exhibit levels of freezing to a trace CS that is comparable to that seen to a delay CS (e.g. [1]). In our lab, such an equivalence of conditioned responding is not observed at other ages, younger or older $[1,36,82]$. Although somewhat perplexing, we have capitalized on this equivalence of conditioned freezing for our studies of alcohol exposure. One argument about alcohol's effects is that it may impact learning of more difficult tasks [3]. In other words, alcohol will have its greatest effects when conditioned responding is weak (but see [95]). With Delay and Trace conditioning procedures, trace conditioning typically results in weaker responding than delay. Thus, it could be argued that a relatively selective alcohol effect on trace conditioning reflects weaker learning or weaker responding. While we are not in a position to conclude equivalent baseline learning across the two learning paradigms in the adolescent animals, the argument that alcohol affects weaker responding can be ruled out since we have shown responding to be equivalent in delay and trace conditioned adolescents.

\section{Experiment 1}

The goal of Experiment 1 was to replicate our previous observation that neonatal ethanol impairs trace conditioning, but has no effect on delay conditioning, in adolescent rats.

\subsection{Method}

\subsubsection{Subjects}

A total of 63 rats ( $\mathrm{ns}=10-12$ /group) from 22 litters served as subjects. Twelve of these litters provided subjects for the EtOH and Sham groups, and an additional 10 litters were used to derive Unhandled controls.

\subsubsection{Apparatus}

The apparatus as described in the General method section was used.

\subsubsection{Procedure}

On PD 4-9 animals were treated with $5 \mathrm{~g} / \mathrm{kg} /$ day ethanol (group EtOH), given sham intubations (group Sham) or were given no treatment during this period (group Unhandled). On PD 30-31 ( \pm 1 day), half of the animals in each neonatal treatment group were trained and tested in the Delay conditioning paradigm and half were trained and tested in the Trace conditioning paradigm. Interest was in the effect of neonatal binge ethanol on hippocampus-dependent trace conditioning.

\subsection{Results $\mathcal{E}$ discussion}

\subsubsection{Body weights}

During the ethanol exposure period (PD 4-9) the EtOH group gained less weight than sham controls. This is typical with this binge model. Body weights recorded during this time in Sham and EtOH animals 
were analyzed using a 2 (neonatal treatment) $\times 2$ (sex) $\times 6$ (day) mixed-factor Analysis of Variance (ANOVA). The ANOVA revealed main effects of neonatal treatment $[F(1,39)=27.40, p<.001]$ and day $[\mathrm{F}(5,195)=839.09, \mathrm{p}<.001]$ and a Neonatal Treatment $\times$ Day interaction $[\mathrm{F}(5,195)=58.11, \mathrm{p}<.001]$. Newman-Keuls post-hoc tests indicated that the groups began to diverge on PD 6 and the EtOH group continued to weigh less than the Sham group through PD 9. Mean $( \pm$ SEM) weights on PD 9 were $21.40 \mathrm{~g}(0.61)$ for group EtOH and $26.74 \mathrm{~g}$ (0.56) for group Sham.

Importantly, body weights were equivalent in the groups on the day of training, PD 30. A 3 (neonatal treatment) $\times 2$ (sex) ANOVA conducted on weights recorded prior to training revealed only a main effect of sex $[\mathrm{F}(1,57)=17.74, \mathrm{p}<.001]$. By PD 30 the EtOH subjects had gained sufficient weight to no longer be distinguishable from control animals. On PD 30 males weighed more ( $\mathrm{M}=137.02 \mathrm{~g}$; $\mathrm{SEM}=3.48$ ) than females $(\mathrm{M}=114.31 \mathrm{~g} ; \mathrm{SEM}=4.12)$.

\subsubsection{Delay and trace conditioning}

Wagner \& Hunt [97] were the first to demonstrate impaired trace fear conditioning in animals exposed to ethanol on PD 4-9. This finding has been replicated in our lab [34,81,82] and by others [17] and the data shown in Fig. 1 illustrate this effect. A 3 (neonatal treatment) $\times 2$ (conditioning) ANOVA conducted on the $\%$ change freezing scores from the test yielded significant main effects of neonatal treatment $[\mathrm{F}(2,57)=6.53, \mathrm{p}<.001]$ and conditioning $[\mathrm{F}(1,57)=$ $7.43, \mathrm{p}<.01$ ] as well as a Neonatal Treatment $\times$ Conditioning interaction $[\mathrm{F}(2,57)=6.62, \mathrm{p}<.01]$. Post-hoc Newman-Keuls tests revealed that ethanol-treated animals were impaired in trace, but not delay, fear conditioning.

The black bars in Fig. 1 show freezing data from groups of subjects trained in the delay procedure. As can be seen, the groups did not differ as a function of neonatal treatment, and all exhibited high levels of CSelicited freezing. The gray bars of Fig. 1 show results from the traceconditioned groups. Unhandled and Sham-treated animals given trace conditioning trials showed high levels of CS-elicited freezing, and the magnitude of the freezing response did not differ significantly from the respective delay-conditioned subjects. However, the ethanoltreated animals failed to show any evidence of trace conditioning. CSelicited freezing was virtually zero revealing that neonatal ethanol

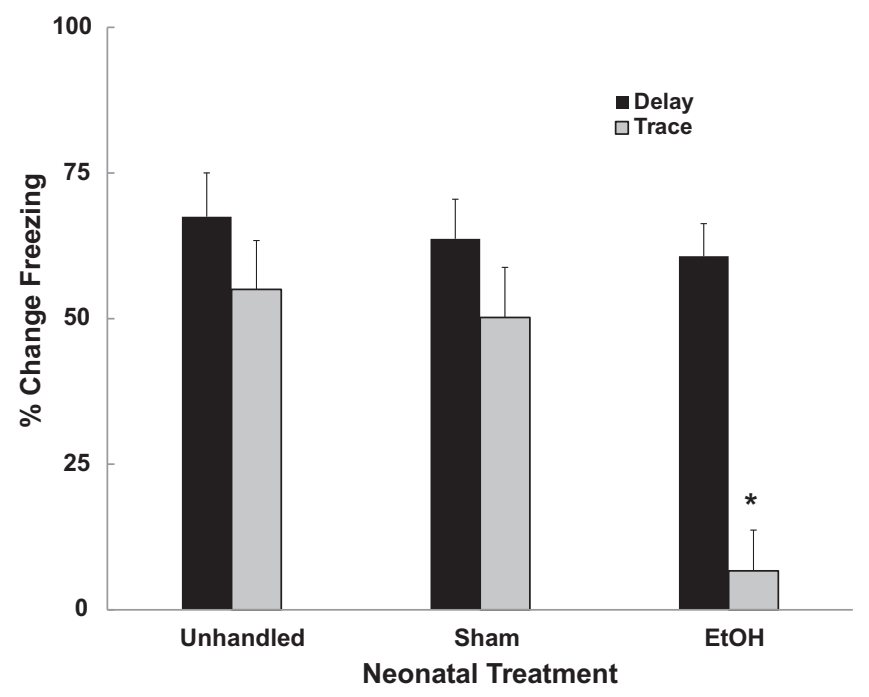

Fig. 1. Mean ( \pm SEM) percent freezing (\% CS freezing - \% pre-CS freezing) to the light CS during the test in Experiment 1. Animals were given CS-US pairings in either a Delay or Trace fashion and CS-elicited freezing was measured $24 \mathrm{~h}$ later. Neonatal groups were Sham intubated, administered $5 \mathrm{~g} / \mathrm{kg} /$ day ethanol (EtOH) or were untreated (Unhandled) prior to training. exposure impaired hippocampus dependent trace conditioning in adolescent animals.

Research reported by Hunt et al. [34] varied a number of aspects of the neonatal ethanol protocol as well as the training procedures. Hunt et al. [34] showed that: (a) the trace conditioning deficit observed in ethanol-treated subjects was dose-dependent; (b) the impairment in trace conditioning resulting from PD 4-9 ethanol exposure depends on the duration of the trace interval, with ethanol-exposed animals performing like controls with shorter intervals, and (c) the effect of PD 4-9 ethanol on trace conditioning was entirely driven by ethanol exposure occurring during the first three days of this period (PD 4-6). Ethanol exposure limited to PD 7-9 had no effect on trace conditioning, with PD 7-9 ethanol-treated subjects displaying a level of trace freezing that was comparable to sham controls. We have also demonstrated that the ethanol-induced impairment in trace conditioning persists into young adulthood in female subjects [82]; females trained and tested as adults (65-70 days of age) continue to perform poorly after trace conditioning training. This finding suggests that neonatal ethanol exposure produces a relatively permanent functional deficit in this task (see also $[17,81]$ ), although these effects are specific to females.

\section{Experiment 2}

Findings from Experiment 1 for Delay and Trace fear conditioning clearly demonstrate that neonatal alcohol exposure does not uniformly affect all types of associative fear conditioning (see also [23,63]). This is important because it suggests some forms of learning may be protected from the damaging effects of fetal alcohol and raises the translational issue of how learning might be promoted to attenuate some of the cognitive deficits associated with alcohol exposure. In Experiment 2 we explored an environmental manipulation that might allow rats to overcome the ethanol-induced impairment in trace conditioning seen in Experiment 1.

Trace conditioning can be facilitated by a variety of means. Increases in conditioned responding to a trace CS are evident with decreasing the length of the trace interval $[9,34,62]$. Another procedure involves inserting a nontarget CS into the trace interval. The latter effect has been characterized as 'gap filling', or serial compound conditioning. Using the serial procedure, subjects are trained with two serially presented CSs, CS1 $\rightarrow$ CS2 $\rightarrow$ US, and performance to the target CS1 is compared to that resulting from standard trace conditioning procedures, CS1 $\rightarrow$ trace interval $\rightarrow$ US. In each case there is an equivalent trace interval between offset of CS1 and onset of the US. However, the presence of CS2 in the serial procedure has been shown to facilitate acquisition to CS1 (see $[19,29,70,75]$ for different explanations of the gap filling effect).

Hunt et al. [32] reported that training with the serial compound (CS1 light $\rightarrow$ CS2 tone $\rightarrow$ US shock) led to a noticeable improvement in learning about the target CS1 light in both PD 18 and PD 25 animals. Subjects trained with the serial compound procedure exhibited significantly more freezing to the light CS1 than groups trained with standard trace conditioning despite equivalent intervals separating offset of CS1 from the US. Several observations in those experiments led to our interest in examining this type of learning in our animal model of FASD.

First, as implied, the serial compound procedure is essentially a trace conditioning procedure, where the trace interval is filled with a nontarget CS. Therefore the serial procedure can be used to further examine conditions under which trace conditioning deficits emerge. Second, Hunt et al. [32] observed virtually no standard trace conditioning (freezing to the target CS) in PD 18 animals and weak to moderate trace conditioning in PD 25 animals. Serial conditioning or gap filling (CS1 $\rightarrow$ CS2 $\rightarrow$ US) markedly enhanced performance in each age group. This finding is important because it suggested that under some conditions cognitive deficits measured in trace conditioning might be overcome by use of the serial procedure. In Hunt et al. [32] those (trace conditioning) deficits were age related and in Experiment 2 of 
the present research they were produced by neonatal ethanol. This leads to the interesting possibility tested in Experiment 2 that the trace conditioning deficit otherwise produced by neonatal ethanol might be overcome by serial compound conditioning.

\subsection{Method}

\subsubsection{Subjects}

The subjects were 76 rats derived from 25 litters (10 Unhandled). Group sizes ranged from 10-13.

\subsubsection{Apparatus}

The apparatus as described in the General method section was used. In addition, CS2 was an $80 \mathrm{~dB} 1600 \mathrm{~Hz}$ pulsing (.25 s ON/.25 s OFF, $250 \mathrm{~ms}$ rise/fall time) tone. During training, the tone was presented through a speaker mounted in the ceiling of the conditioning chamber. During testing in a novel context, the speaker was mounted in the ceiling of the sound-attenuating shell.

\subsubsection{Procedure}

As in Experiment 1, on PD 4-9 Ethanol animals were exposed to $5 \mathrm{~g} / \mathrm{kg} /$ day ethanol, Sham animals were given sham intubations, and Unhandled animals received no treatment. On PD 30, half of the animals in each neonatal condition were trained and tested in the standard trace conditioning paradigm as described in Experiment 1 (CS1 light $\rightarrow$ trace interval $\rightarrow$ US shock) while the other half were trained and tested in the serial conditioning paradigm (CS1 light $\rightarrow$ CS2 tone $\rightarrow$ US shock). Specifically, Trace groups were exposed to a 10-s flashing light (CS1) followed by a trace interval of $10 \mathrm{~s}$ followed by the footshock US. In the serial compound procedure, the 10-s flashing light (CS1) was followed by a 10-s pulsing tone (CS2) and the tone was immediately followed by the footshock US. Animals in the Trace condition were additionally exposed to an equal number of random presentations of the 10 -s tone, in order to equate exposure to the tone CS in Trace groups with that of the Serial groups. All subjects were then tested for freezing to both the light and tone. Animals were tested for freezing to the light $24 \mathrm{~h}$ after training, and tested for freezing to the tone $24 \mathrm{~h}$ after the light test.

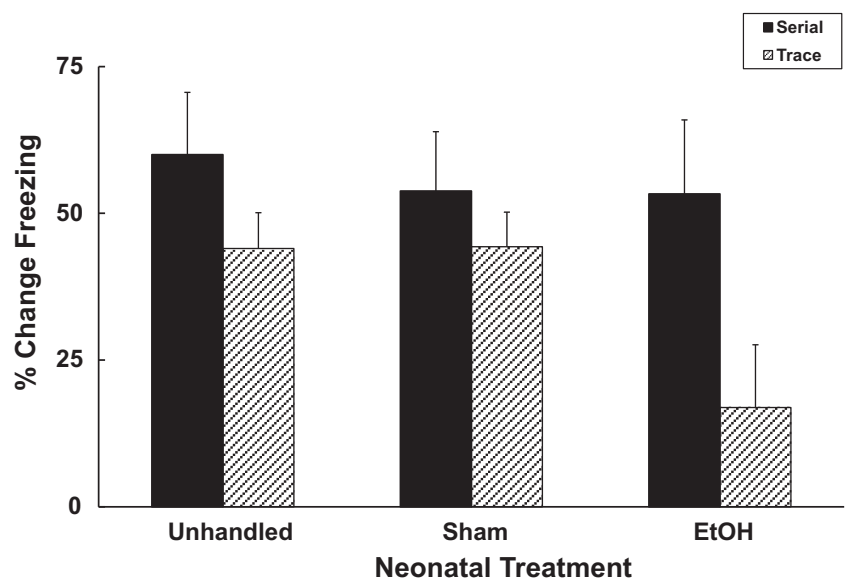

Fig. 2. Mean ( \pm SEM) percent freezing (\% CS freezing - \% pre-CS freezing) to the light CS during the test in Experiment 2. During the neonatal period (postnatal days 4-9) animals were given Sham intubations, were administered $5 \mathrm{~g} / \mathrm{kg} /$ day ethanol $(\mathrm{EtOH})$ or were untreated (Unhandled). Training on day 30 involved serial presentations of the CSs (light $\rightarrow$ tone $\rightarrow$ shock) or trace conditioning (light $\rightarrow$ trace interval $\rightarrow$ shock) with random presentations of the tone.

\subsection{Results $\mathcal{E}$ discussion}

Fig. 2 shows the results of trace and serial compound conditioning on responding to the light (CS1). Analyses of freezing scores yielded a main effect of training $[\mathrm{F}(1,70)=8.75, \mathrm{p}<.01]$. Training with a serial compound enhanced trace conditioning in all groups, although the effect is particularly pronounced in the EtOH group. Moreover, there was no evidence of a trace conditioning deficit to the target CS1 in serially trained animals that received neonatal ethanol exposure. Serial conditioning reversed the ethanol-induced deficit in trace conditioning. Responding to the tone (CS2) was robust in the Serial groups compared with the Trace groups that received random presentations of CS2 [main effect of training, $F(1,70)=27.87, p<.001]$. Further, there were no differences among the neonatal treatment groups $[\mathrm{F}<1]$. Mean freezing scores $( \pm$ SEM $)$ from the tone test for groups EtOH-Serial, Sham-Serial and Unhandled-Serial were 55.7\% (8.8), 50.0\% (7.4) and 51.6\% (9.3), respectively. The average freezing response to the tone in Trace groups was $8.4 \%$ (5.6).

The results from Experiment 2 parallel those from Experiment 1 in an important way. Both experiments reveal that neonatal ethanol does not impair learning in all circumstances. Results of Experiment 1 demonstrated ethanol-induced impairments in trace but not delay conditioning. Experiment 2 revealed deficits in standard trace conditioning but not serial trace conditioning despite neonatal ethanol exposure and equivalent trace intervals between the target CS1 and US. This latter finding with the serial procedure is important. It suggest some environmental manipulations that enhance learning are less subject or less likely to be undermined despite the impairing effects of perinatal alcohol exposure. In the present experiment, the cognitive enhancing effect of serial conditioning on learning to a trace CS demonstrated just such a resilience. Developing environmental treatments (in addition to pharmacological interventions as explored later) that reduce cognitive deficits from neonatal alcohol exposure may be particularly valuable given the possibility that the effectiveness of some pharmacological interventions may be marginalized by permanent neurophysiological effects of fetal alcohol that precede intervention attempts.

\section{Nutritional and pharmacological treatments targeting the cholin- ergic system}

Since the initial reports of FAS [40], efforts at reducing or eliminating maternal drinking have been in place. Unfortunately, these have been mostly unsuccessful. Estimates of the incidence of FAS today are the same as they were in the 1970s. More effective methods for promoting abstinence during pregnancy are sorely needed, but until that time research is targeting treatments for the afflicted individuals. Below we describe data from two experiments that examined specific nutritional (choline) and pharmacological (physostigmine) interventions for the short-term memory impairment seen in trace conditioning after ethanol exposure.

\subsection{Supplemental choline}

Work from several research labs (e.g. $[7,58,99]$ ) has yielded important information about the role of dietary choline on brain function and behavior. Supplementation of the diet with additional choline during pre- and early post-natal development can lead to the earlier onset of function in hippocampal and frontal cortical systems [58], and can even delay age-related cognitive decline [22]. Although the mechanisms by which choline acts are not clearly understood, choline seems to support the development of stronger cholinergic systems that are less vulnerable to a variety of insults [26]. Choline serves many biological functions, including being a precursor of the structural phospholipids phosphatidylcholine and sphingomyelin, and signaling lipids [4]. Choline is the precursor for acetylcholine, which not only serves a neurotransmitter function but also is an important developmental growth 
factor [57]. Meck and Williams [58] suggest that supplemental choline early in life may promote the tuning of cellular networks in the brain, especially cholinergic networks.

Thomas and colleagues have extensively examined the beneficial effects of supplemental choline in a rat model of FASD. Supplementation with choline during and/or after the ethanol exposure period can significantly attenuate many adverse effects of ethanol, especially in hippocampus-dependent memory tasks. For example, Thomas et al. [89] reported that choline administered on PD 2-21 reversed the deficits in visual discrimination learning resulting from prenatal ethanol exposure. Ryan et al. [78] found that neonatal ethanol (PD 4-9) resulted in impaired performance in the Morris water maze, and that choline administered on PD 11-30 attenuated this effect. Several other findings from this lab highlight the beneficial effects of supplemental choline as a potential treatment for FASD (e.g. [88,90,92]).

Wagner and Hunt [97] used a variant of Thomas' choline supplementation procedure [90] to examine whether choline would effectively mitigate our observed ethanol-induced trace conditioning deficit. Animals were exposed to $5 \mathrm{~g} / \mathrm{kg} / \mathrm{day}$ ethanol or sham intubations on PD 4-9. Half of the animals in each group were given once daily subcutaneous injection of choline chloride $(0.1 \mathrm{ml}$ of an $18.8 \mathrm{mg} / \mathrm{ml}$ solution $)$ and the other half were administered saline. Choline injections began on the first day of ethanol exposure (PD 4) and continued through PD 20. On PD 30-31 subjects were trained and tested in delay or trace conditioning procedures.

Fig. 3 shows some of the key results from Wagner and Hunt [97]. The data indicate that choline supplementation completely reversed the ethanol deficit in trace fear conditioning. A 2 (neonatal treatment) $\times 2$ (choline dose) ANOVA revealed a main effect of choline dose [F ( 1 , $39)=5.03, \mathrm{p}=.031$ ] and, importantly, a significant neonatal treatment $\times$ choline dose interaction $[F(1,39)=6.19, p=.017]$. Post-hoc Newman-Keuls tests support the conclusions that animals administered ethanol were severely impaired in trace fear conditioning (group EtOH-Saline), but those treated with supplemental choline during and after the ethanol exposure period (group EtOH-Choline) responded with the same level of CS-elicited freezing as Sham controls. Choline administration had no effect on delay conditioned responding in either neonatal treatment group (see [97]). Notably, Thomas and Tran [92] have reported an identical effect using a trace eyeblink conditioning procedure in adolescent rats. Similarly to our fear conditioning data,

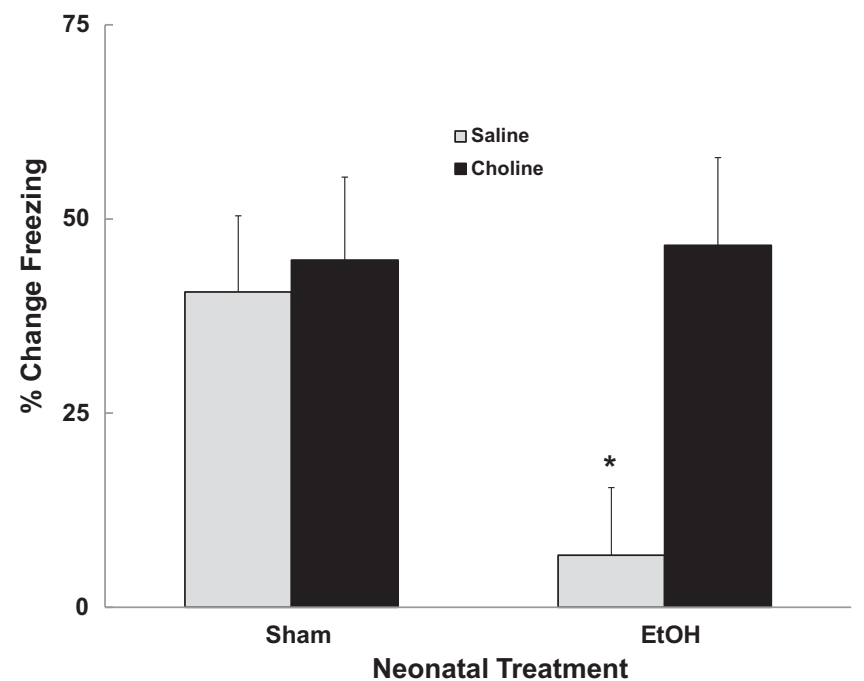

Fig. 3. Mean ( \pm SEM) percent freezing (\% CS freezing - \% pre-CS freezing) to a trace-conditioned CS in animals given supplemental choline. On postnatal days 4-9, animals were given Sham intubations, or administered $5.25 \mathrm{~g} / \mathrm{kg} / \mathrm{day}$ ethanol (EtOH). Beginning on postnatal day 4 and continuing through day 20 , animals were given an sc injection of saline or choline chloride. On day 30 , animals were trained with trace CS-US pairings, and tested for CS-elicited freezing $24 \mathrm{~h}$ later. (From [97]). choline supplementation had a beneficial effect on trace eyeblink conditioning, which was impaired by neonatal ethanol exposure, but no effect on delay eyeblink conditioning. Whether the benefits of supplemental choline on trace conditioning result from its preventing alcoholinduced damage (given during the ethanol administration procedure) and/or reversing the effects of ethanol (given after ethanol administration) remains to be determined, although some data [78,89] suggest that choline supplementation can be beneficial even when provided after the ethanol exposure period consistent with a restorative function.

\subsection{The cholinesterase inhibitor, physostigmine}

Mild cognitive impairments, including those seen in early stage Alzheimer's Disease, are oftentimes treated with cholinesterase inhibitors [15]. This is the case when the cognitive impairments relate to memory that is cholinergic. Given that the cholinergic system, including both nicotinic and muscarinic receptor subtypes, is necessary for trace conditioning $[36,42,74]$, here we reasoned that trace conditioning in ethanolexposed animal would be improved by pre-training administration of the cholinesterase inhibitor physostigmine. These cholinesterase inhibitors should be strong candidates for treatment in our ethanol-induced trace conditioning impairments, since trace conditioning is cholinergically mediated.

This was a preliminary study in which animals ( $n=6 /$ group) were given $5 \mathrm{~g} / \mathrm{kg} /$ day ethanol or sham intubations on PD 4-9. On PD 30-31 animals were trained with our standard trace fear conditioning procedure and tested $24 \mathrm{~h}$ later. Animals in the EtOH and Sham groups were injected intraperitoneally with one of several doses of physostigmine $(0,0.001,0.005$ or $0.01 \mathrm{mg} / \mathrm{kg}) 10 \mathrm{~min}$ prior to conditioning. Animals were tested for CS-elicited freezing $24 \mathrm{~h}$ later in a drug-free state. Our preliminary results are shown in Fig. 4.

Pre-training administration of physostigmine produced a dosedependent improvement in trace conditioning in ethanol-treated animals, with the highest dose having the greatest effect. The Shamtreated subjects, however, exhibited no improvement in trace learning at any dose of physostigmine. This is in contrast to previous data

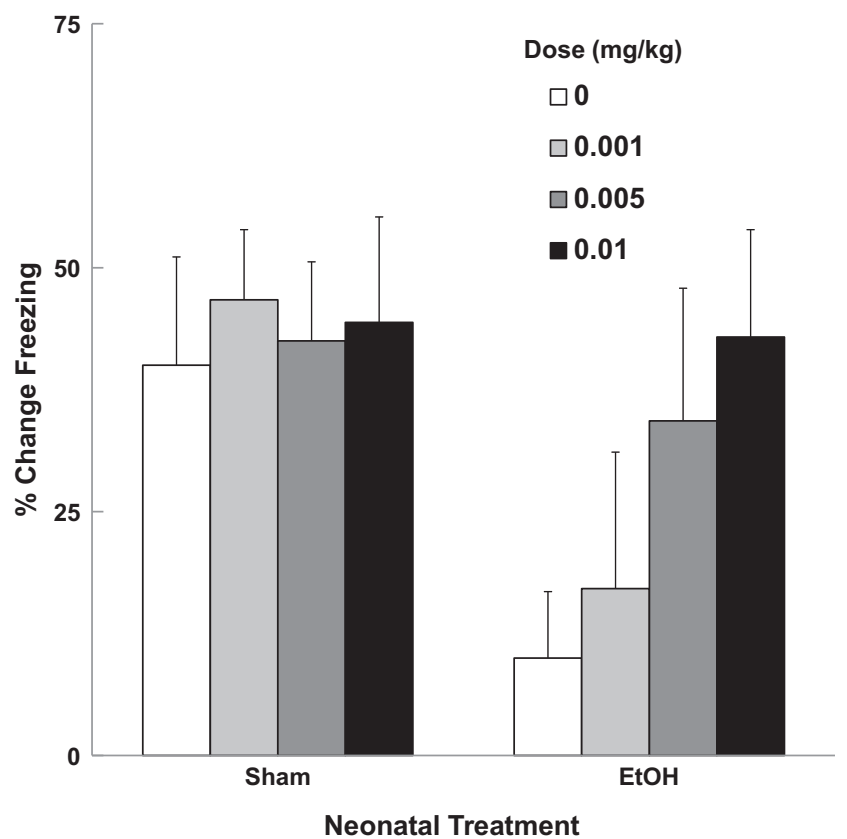

Fig. 4. Mean percent freezing (\% CS freezing - \% pre-CS freezing) in subjects given pretraining physostigmine injections. On postnatal days 4-9 animals were given Sham intubations or $5 \mathrm{~g} / \mathrm{kg} /$ day ethanol (EtOH). Subjects were given trace conditioning trials on day 30, and tested for CS-elicited freezing $24 \mathrm{~h}$ later. Animals were injected ip with one of several doses of physostigmine $(0,0.001,0.005$ or $0.01 \mathrm{mg} / \mathrm{kg}) 10 \mathrm{~min}$ prior to training. No drug was given prior to test. 
published by Hunt \& Richardson [36]. In that paper we reported that trace conditioning in experimentally-naïve subjects was enhanced by the same pre-training physostigmine treatment. One major difference between that study and the present was the level of conditioned responding in controls. Hunt and Richardson tested 25-day-old animals which typically show low levels of conditioned freezing with these trace procedures (see also [1,61]). With modest levels of conditioned freezing, an enhancement by physostigmine was observed. The data in Fig. 4 is from animals 30 days of age, which in our lab typically show maximal levels of freezing (see Section 2.3.4). Thus, a ceiling effect on levels of freezing in control animals may be present, precluding observation of an enhancement due to physostigmine.

\section{Summary and conclusions}

Animal research has verified that ethanol is a teratogenic agent, and that the hippocampus is one of the neural regions that is particularly vulnerable to ethanol toxicity. A number of reports have revealed that the number and density of pyramidal neurons in areas CA1 and CA3 are reduced following perinatal exposure $[5,25,49,94]$. Reductions in numbers of granule cells in the dentate gyrus have also been observed ([49]; but see [5]). The trace conditioning paradigm is a particularly elegant procedure for clarifying the functional (i.e. cognitive and behavioral) consequences of these kinds of documented neural alterations produced by neonatal alcohol. The neuroanatomical and neurochemical substrates for trace and delay fear conditioning are quite well characterized (e.g. $[48,73,87])$. Animals trained with trace or delay CS-US pairings are treated identically during training, except for the duration of the trace interval. Subjects are exposed to the same CS, the same US, and the same experimental context during training; the only difference is that one group is trained with a stimulus-free period separating CS offset from US onset. During the test, performance is evaluated under identical stimulus presentations. Thus, differences in performance during the test are only attributable to the differences in training parameters.

A key finding from the present experiments was that neonatal ethanol exposure impaired hippocampus dependent trace conditioning. In Experiment 1 ethanol impaired trace conditioning (CS1 $\rightarrow$ trace interval $\rightarrow$ US) relative to sham intubated and unhandled controls. No such impairment was observed in delay conditioning. Examination of the effects of neonatal ethanol exposure in the serial procedure $(\mathrm{CS} 1 \rightarrow \mathrm{CS} 2 \rightarrow \mathrm{US})$ of Experiment 2 additionally suggested that different types of learning processes (i.e., associative structures) are not equally susceptible to the damaging effects of ethanol exposure. Various explanations for the gap filling effect in the serial procedure have been proposed including one type of higher-order conditioning known as second-order conditioning [43]. This view posits that the animals acquire fear to the non-target CS2 because it is proximally followed by the shock US in the serial procedure ( $\mathrm{CS} 1 \rightarrow \mathrm{CS} 2 \rightarrow \mathrm{US})$. Subsequently, fear to the target CS1 is acquired through its association with CS2 which serves as a conditioned reinforcer. A different kind of higherorder conditioning called sensory pre-conditioning proposes that animals acquire an association between the CS2 and CS1, and then one of the elements (e.g., CS2) is associated with the shock US. In related experiments (Barnet \& Hunt, unpublished data) we observed that alcohol-exposed animals exhibit normal second-order conditioning, but are impaired in sensory pre-conditioning. The point of connection of these findings to those of Experiment 2 is that some environmental interventions which promote the expression of otherwise absent (or behaviorally silent) learning may be more capable than others at protecting against the detrimental consequences of early alcohol exposure. Furthermore, given that second-order conditioning and sensory preconditioning have been suggested to be mediated by different associative structures [77], these recent findings also suggest some learning processes themselves may be more resilient to the damaging effects of early alcohol exposure. Taken together with the results of Experiment 2 it becomes clear that some environmental interventions may be better than others in overcoming the cognitive deficits associated with neonatal ethanol exposure.

Because efforts to prevent maternal alcohol use during pregnancy have been only modestly successful, further efforts to treat the already afflicted individual have become a major focus of study. Several 'environmental' intervention programs (e.g., cognitive and behavioral training) have been described for young children and adolescents, and many produce beneficial effects $[2,8,41]$. These interventions typically target a specific behavior or skill, such as attention dysregulation, social skills or verbal abilities. Research with animal subjects has studied treatments and interventions in well-controlled models of FASD. Much of this research has focused on the hippocampus and hippocampus-dependent memory impairments in alcohol-exposed rodents.

Results of Wagner \& Hunt [97] suggest supplemental choline as an intervention or treatment for ethanol-induced memory impairment. Our findings indicate that supplementation with choline during and for a brief time after ethanol exposure can completely reverse the ethanol-induced deficit in hippocampus-dependent trace conditioning (Fig. 3). The extensive work from Thomas' lab has revealed that many other learning impairments are benefitted from choline. For example, Thomas and colleagues $[78,89]$ have shown that choline administration that occurs after the ethanol exposure period can reverse ethanol's toxic effects on hippocampus dependent spatial learning. Moreover, supplementation with choline has been shown to prevent or reverse performance deficits in hippocampus-, as well as frontal cortex-dependent tasks $[88,89]$. Finally, other nutritional supplements, such as zinc, can increase fetal survival and reduce the physical abnormalities associated with fetal alcohol exposure [85]. From a translational perspective, these findings are extremely important.

While choline supplementation has rather consistently been found effective for treating hippocampus-dependent learning impairments, it is not a treatment for all of ethanol's deleterious effects. Thomas et al. [90] reported no beneficial effect of supplemental choline on motor coordination deficits resulting from neonatal ethanol exposure. Thomas and Tran [92] showed that choline benefits trace eyeblink conditioning, but has no effect on delay eyeblink conditioning, which was also compromised in ethanol-exposed animals. In our lab, choline supplementation had no effect on another type of short-term memory, habituation of the heart rate orienting response to a novel olfactory stimulus [33].

Cognitive enhancers (cholinesterase inhibitors, memantine; [15,37]) can also improve performance on hippocampal tasks. A preliminary study described above (Fig. 4) suggests that the cholinesterase inhibitor physiostimine reduced the ethanol mediated impairment in trace conditioning in a dose-dependent manner. Other promising treatment interventions have also been explored. Antioxidants (Vitamin E, $\beta$ carotine, resveratrol) given at the time of alcohol exposure may prevent alcohol-induced hippocampal cell loss and deficits in spatial memory performance $[12,55,93]$. Behavioral interventions such as voluntary exercise and long-term exposure to complex environments can increase hippocampal neurogenesis (G.F. [28]) and improve performance on hippocampus-dependent tasks (e.g. [91]).

Here we have shown that an environmental manipulation (serial compound training), a nutritional supplement (choline), and a pharmacological treatment (cholinesterase inhibitor) can all effectively mitigate deficits in trace conditioning caused by neonatal ethanol exposure. But not all interventions are effective in reversing the trace conditioning impairment. Schreiber et al. [81] examined the potential benefits of exercise and environmental complexity. In this study animals were treated with ethanol on PD 4-9 or given sham intubations. Beginning on PD 31 the animals were given free access to a running wheel for 12 days, followed by extended rearing in a complex environment (PD 42-72). Animals were then trained and tested in trace conditioning beginning on PD 82. The exercise and complex environment manipulation had no beneficial effect on trace conditioning; these animals showed the same deficit in trace conditioning as those reared 
under standard housing conditions. Collectively, the results of numerous studies indicate that while many treatments and interventions are effective for specific neural or behavioral dysfunction, no one intervention or therapy is sufficient to treat all features of FASD. Advances in treatment which are most profitable are likely those to be informed by research which characterizes, specifically, the nature of ethanolinduced impairments, and that informs correspondingly specific intervention.

\section{Acknowledgments}

The research presented in this paper was supported by NIH grant AA015343, a grant from the Virginia Tobacco Settlement Foundation, a Howard Hughes Medical Institute grant through the Undergraduate Biological Sciences Education Program to the College of William \& Mary, and student support through the Roy R. Charles Center at the College of William \& Mary. The authors wish to thank the many undergraduate students who assisted with data collection.

\section{References}

[1] R.C. Barnet, P.S. Hunt, Trace and long-delay fear conditioning in the developing rat, Learn Behav 33 (2005) 437-443.

[2] J. Bertrand, Interventions for children with fetal alcohol spectrum disorders (FASADs): overview of findings for five innovative research projects, Res Dev Disabil 30 (2009) 986-1006

[3] A.V. Beylin, C.C. Gandhi, G.E. Wood, A.C. Talk, L.D. Matzel, T.J. Shors, The role of the hippocampus in trace conditioning: temporal discontinuity or task difficulty? Neurobiol Learn Mem 76 (2001) 447-461.

[4] J.K. Blusztajn, J.M. Cermak, T. Holler, D.A. Jackson, Imprinting of hippocampal metabolism of choline by its availability during gestation: implications for cholinergic neurotransmission, J Physiol 92 (1998) 199-203.

[5] D.J. Bonthius, J.R. West, Alcohol-induced neuronal loss in developing rats: increased brain damage with binge exposure, Alcohol Clin Exp Res 14 (1990) 107-118.

[6] R.T. Brown, C.D. Coles, I.E. Smith, K.A. Platzman, J. Silverstein, S. Erickson, et al., Effects of prenatal alcohol at school age. II. Attention and behavior, Neurotoxicol Teratol 13 (1991) 369-376.

[7] J.M. Cermak, T. Holler, D.A. Jackson, J.K. Blusztajn, Prenatal availability of choline modifies development of the hippocampal cholinergic system, FASEB J 12 (1998) 349-357.

[8] A.N. Chandrasena, R.A.S. Mukherjee, J. Turk, Fetal alcohol spectrum disorders: an overview of interventions for affected individuals, Child Adolesc Mental Health 14 (2009) 162-167.

[9] N. Chowdhury, J.J. Quinn, M.S. Fanselow, Dorsal hippocampal involvement in trace fear conditioning with long, but not short, trace intervals in mice, Behav Neurosc 119 (2005) 1396-1402.

[10] R.E. Clark, J.R. Manns, L.R. Squire, Trace and delay eyeblink conditioning: contrasting phenomena of declarative and nondeclarative memory, Psychol Sci 12 (2001) 304-308.

11] K.M. Clements, T.A. Girard, C.G. Ellard, P.E. Wainwright, Short-term memory impairment and reduced hippocampal c-Fos expression in an animal model of fetal alcohol syndrome, Alcohol Clin Exp Res 29 (2005) 1049-1059.

12] R. Cohen-Karem, G. Koren, Antioxidants and fetal protection against ethanol teratogenicity I. Review of the experimental data and implications to humans, Neurotoxicol Teratol 25 (2003) 1-9.

[13] C.D. Coles, F.C. Goldstein, M.E. Lynch, X. Chen, J.A. Kable, K.C. Johnson, et al., Memory and brain volume in adults prenatally exposed to alcohol, Brain Cogn 75 (2011) 67-77.

[14] K.M. Davis, K.R. Gagnier, T.E. Moore, M. Todorow, Cognitive aspects of fetal alcohol spectrum disorder, WIREs Cogn Sci 4 (2013) 81-92.

[15] J.F. Disterhoft, M.M. Oh, Modulation of cholinergic transmission enhanced excitability of hippocampal pyramidal neurons and ameliorates learning impairments in aging animals, Neurobiol Learn Mem 80 (2003) 223-233.

[16] J. Dobbing, J. Sands, Comparative aspects of the brain growth spurt, Early Hum Dev 3 (1979) 79-83.

[17] C.M. DuPont, J.J. Coppola, R.M. Kaercher, D.H. Lindquist, Impaired trace fear conditioning and diminished ERK1/2 phosphorylation in the dorsal hippocampus of adult rats administered alcohol as neonates, Behav Neurosci 128 (2014) 187-198.

[18] M.S. Fanselow, Conditional and unconditional components of post-shock freezing, Pavlov J Biol Sci 15 (1980) 177-182.

[19] C.M. Gibbs, E.J. Kehoe, I. Gormezano, Conditioniong of the rabbit's nictitating membrane response to a CSA-CSB-US serial compound: Manipulations of CSB's associative character, J Exp Psychol Anim Behav Process 17 (1991) 423-432.

[20] J. Gil-Mohapel, F. Boehme, L. Kainer, B.R. Christie, Hippocampal cell loss an neurogenesis after fetal alcohol exposure: insights from different rodent models, Brain Res Rev 64 (2010) 283-303.

[21] T.A. Girard, H.-C. Xing, G.R. Ward, P.E. Wainwright, Early postnatal alcohol exposure has long-term effects on the performance of male rats in a delayed matching-toplace task in the Morris water maze, Alcohol Clin Exp Res 24 (2000) 300-306.
[22] M.J. Glenn, E.D. Kirby, E.M. Gibson, S.J. Wong-Goodrich, T.J. Mellott, J.K. Blusztajn, et al., Age-related declines in exploratory behavior and markers of hippocampal plasticity are attenuated by prenatal choline supplementation in rats, Brain Res 1237 (2008) $110-123$.

[23] M.J. Goodfellow, D.H. Lindquist, Significant long-term, but not short-term, hippocampal-dependent memory impairment in adult rats exposed to alcohol in early postnatal life, Dev Psychobiol (2014), http://dx.doi.org/10.1002/dev.21210.

[24] C.R. Goodlett, T. B. Johnson, Neonatal binge ethanol exposure using intubation: timing and dose effects on place learning, Neurotoxicol Teratol 19 (1997) 435-446.

[25] P.L. Greene, J.L. Diaz-Granados, A. Amsel, Blood ethanol concentration from early postnatal exposure: effects on memory-based learning and hippocampal neuroanatomy in infant and adult rats, Behav Neurosci 106 (1992) 51-61.

[26] S.X. Guo-Ross, S. Clark, D.A. Montoya, K.H. Jones, J. Obernier, A.K. Shetty, et al., Prenatal choline supplementation protects against postnatal neurotoxins, J Neurosci 22 (2002) RC195.

[27] D.A. Hamilton, P. Kodituwakku, R.J. Sutherland, D.D. Savage, Children with fetal alcohol syndrome are impaired at place learning but not cued-navigation in a virtual Morris water task, Behav Brain Res 143 (2003) 85-94.

[28] G.F. Hamilton, K.E. Boschen, C.R. Goodlett, W.T. Greenough, A.Y. Klintsova, Housing in environmental complexity following wheel running augments the survival of newly generated hippocampal neurons in a rat model of binge alcohol exposure during the third trimester equivalent, Alcohol Clin Exp Res 36 (2012) 1196-1204.

[29] P.C. Holland, R.T. Ross, Within-compound associations in serial compound conditioning, J Exp Psychol Anim Behav Process 7 (1981) 228-241.

[30] R.R. Holson, B. Pearce, Principles and pitfalls in the analysis of prenatal treatment effects in multiparous species, Neurotoxicol Teratol 14 (1992) 221-228.

[31] H.E. Hoyme, P.A. May, W.O. Kalberg, P. Kodituwakku, J.P. Gossage, P.M. Trujillo, et al., A practical clinical approach to diagnosis of fetal alcohol spectrum disorders: clarification of the 1996 Institute of Medicine criteria, Pediatrics 115 (2005) 39-47.

[32] P.S. Hunt, R.C. Barnet, M.E. Shea, E.M. Baker, Cholinergic modulation of trace conditioning trained in serial compound: a developmental analysis, Neurobiol Learn Mem 86 (2006) 311-321.

[33] P.S. Hunt, S.E. Jacobson, S. Kim, Supplemental choline does not attenuate the effects of neonatal ethanol administration on habituation of the heart rate orienting response, Neurotoxicol Teratol 44 (2014) 121-125.

[34] P.S. Hunt, S.E. Jacobson, E.J. Torok, A rat model of fetal alcohol exposure exhibits deficits in trace fear conditioning: dose-response and timing effects, Alcohol 43 (2009) 465-474.

[35] P.S. Hunt, J.S. Phillips, Postnatal binge ethanol exposure affects habituation of the cardiac orienting response to an olfactory stimulus in preweanling rats, Alcohol Clin Exp Res 28 (2004) 123-130.

[36] P.S. Hunt, R. Richardson, Pharmacological dissociation of trace and long delay fear conditioning in young rats, Neurobiol Learn Mem 87 (2007) 86-92.

[37] N.M. Idrus, N.N.H. McGough, M.J. Spinetta, J.D. Thomas, E.P. Riley, The effects of a single memantine treatment on behavioral alterations associated with binge alcohol exposure in neonatal rats, Neurotoxicol Teratol 33 (2011) 444-450.

[38] D. Ivkovich, C.M. Paczkowski, M.E. Stanton, Ontogeny of delay versus trace eyeblink conditioning in the rat, Dev Psychobiol 36 (2000) 148-160.

[39] D. Ivkovich, M.E. Stanton, Effects of early hippocampal lesions on trace, delay, and long-delay eyeblink conditioning in developing rats, Neurobiol Learn Mem 76 (2001) 426-446.

[40] K.L. Jones, D.W. Smith, Recognition of the fetal alcohol syndrome in early infancy, Lancet 2 (1973) 999-1001.

[41] J.A. Kable, C.D. Coles, E. Taddeo, Socio-cognitive habilitation using the math interactive learning experience program for alcohol-affected children, Alcohol Clin Exp Res 31 (2007) 1425-1434.

[42] T. Kaneko, R.F. Thompson, Disruption of trace conditioning of the nictitating membrane response in rabbits by central cholinergic blockade, Psychopharmacology (Berl) 131 (1997) 161-166.

[43] P.S. Kaplan, E. Hearst, Bridging temporal gaps between CS and US in autoshaping: Insertion of other stimuli before, during, and after CS, J Exp Psychol Anim Behav Process 8 (1982) 187-203.

[44] S.J. Kelly, C.R. Goodlett, J.H. Hannigan, Animal models of Fetal Alcohol Spectrum Disorders: impact of the social environment, Dev Disabil Res Rev 15 (2009) 200-208.

[45] P.W. Kodituwakku, Neurocognitive profile in children with Fetal Alcohol Spectrum Disorders, Dev Disabil Res Rev 15 (2009) 218-224.

[46] P.W. Kodituwakku, A neurodevelopmental framework for the development of interventions for children with fetal alcohol spectrum disorders, Alcohol 44 (2010) 717-728.

[47] M. Korkman, S. Kettunen, I. Autti-Ramo, Neurocognitive impairment in early adolescence following prenatal alcohol exposure of varying duration, Child Neuropsychol 9 (2003) 117-128.

[48] J.E. LeDoux, Emotion circuits in the brain, Annu Rev Neurosci 23 (2000) 155-184.

[49] D.J. Livy, E.K. Miller, S.E. Maier, J.R. West, Fetal alcohol exposure and temporal vulnerability: effects of binge-like alcohol exposure on the developing rat hippocampus, Neurotoxicol Teratol 25 (2003) 447-458.

[50] S.N. Mattson, A.M. Goodman, C. Caine, D.C. Delis, E.P. Riley, Executive functioning in children with heavy prenatal alcohol exposure, Alcohol Clin Exp Res 23 (1999) 1808-1815.

[51] S.N. Mattson, E.P. Riley, A review of the neurobehavioral deficits in children with fetal alcohol syndrome or prenatal exposure to alcohol, Alcohol Clin Exp Res 22 (1998) 279-294.

[52] S.N. Mattson, E.P. Riley, Implicit and explicit memory functioning in children with heavy prenatal alcohol exposure, J Int Neuropsychol Soc 5 (1999) 462-471.

[53] S.N. Mattson, E.P. Riley, The quest for a neurobehavioral profile of heavy prenatal alcohol exposure, Alcohol Res Health 34 (2011) 51-55. 
[54] S.N. Mattson, E.P. Riley, L. Gramling, D.C. Dellis, K.L. Jones, Neuropsychological comparison of alcohol-exposed children with or without physical features of fetal alcohol syndrome, Neuropsychology 12 (1998) 146-153.

[55] M.D. Marino, M.Y. Aksenov, S.J. Kelly, Vitamin E protects against alcohol-induced cell loss and oxidative stress in the neonatal rat hippocampus, Int J Dev Neurosci 22 (2004) 363-377.

[56] P.A. May, J.P. Gossage, Estimating the prevalence of fetal alcohol syndrome: a summary, Alcohol Res Health 25 (2001) 159-167.

[57] J.C. McCann, M. Hudes, B.N. Ames, An overview of evidence for a causal relationship between dietary availability of choline during development and cognitive function in offspring, Neurosci Biobehav Rev 30 (2006) 696-712.

[58] W.H. Meck, C.L. Williams, Metabolic imprinting of choline by its availability during gestation: implications for memory and attentional processing across the lifespan, Neurosci Biobehav Rev 27 (2003) 385-399.

[59] B.R. Monk, F.M. Leslie, J.D. Thomas, The effects of perinatal choline supplementation on hippocampal cholinergic development in rats exposed to alcohol during the brain growth spurt, Hippocampus 22 (2012) 1750-1757.

[60] K.C. Morasch, P.S. Hunt, Persistent deficits in orienting response habituation following neonatal binge ethanol exposure, Alcohol Clin Exp Res 33 (2009) 1596-1604.

[61] T.B. Moye, J.W. Rudy, Ontogenesis of trace conditioning in young rats: dissociation of associative and memory processes, Dev Psychobiol 20 (1987) 405-414.

[62] J.R. Moyer, R.A. Deyo, J.F. Disterhoft, Hippocampectomy disrupts trace eyeblink conditioning in rabbits, Behav Neurosci 104 (1990) 243-252.

[63] N.J. Murawski, M.E. Stanton, Variants of contextual fear conditioning are differentially impaired in the juvenile rat by binge ethanol exposure on postnatal days 4-9, Behav Brain Res 212 (2010) 133-142.

[64] N.J. Murawski, M.E. Stanton, Effects of dose and period of neonatal alcohol exposure on the context preexposure facilitation effect, Alcohol Clin Exp Res 35 (2011) 1160-1170.

[65] A.H. Nagahara, R.J. Handa, Fetal alcohol exposure produces delay-dependent memory deficits in juvenile and adult rats, Alcohol Clin Exp Res 21 (1997) 710-715.

[66] A.H. Nagahara, R.J. Handa, Fetal alcohol-exposed rats exhibit differential response to cholinergic drugs on a delay-dependent memory task, Neurobiol Learn Mem 72 (1999) 230-243.

[67] A. Niccols, Fetal alcohol syndrome and the developing socio-emotional brain, Brain Cogn 65 (2007) 135-142.

[68] E. Nio, K. Kogure, T. Yae, H. Onodera, The effects of maternal ethanol exposure on neurotransmission and second messenger systems: a quantitative autoradiographic study in the rat brain, Dev Brain Res 62 (1991) 51-60.

[69] H.C. Olson, J.J. Feldman, A.P. Streissguth, P.D. Sampson, F.L. Bookstein, Neuropsychological deficits in adolescents with fetal alcohol syndrome: clinical findings, Alcohol Clin Exp Res 22 (1998) 1998-2012.

[70] J.M. Pearce, D.J. Nichols, A. Dickinson, The potentiation effect during serial conditioning, Q J Exp Psychol 33B (1981) 159-179.

[71] D.R. Pierce, D.C. Serbus, K.E. Light, Intragastric administration of alcohol during postnatal development of rats results in selective cell loss in the cerebellum, Alcohol Clin Exp Res 17 (1993) 1275-1280.

[72] M.P. Puglia, C.F. Valenzuela, Repeated third trimester-equivalent ethanol exposure inhibits long-term potentiation in the hippocampal CA1 region of neonatal rats, $\mathrm{Al}$ cohol 44 (2010) 283-290.

[73] J.J. Quinn, S.S. Oommen, G.E. Morrison, M.S. Fanselow, Post-training excitotoxic lesions of the dorsal hippocampus attenuate forward trace, backward trace, and delay fear conditioning in a temporally specific manner, Hippocampus 12 (2002) 495-504.

[74] J.D. Raybuck, T.J. Gould, The role of nicotinic acetylcholine receptors in the medial prefrontal cortex and hippocampus in trace fear conditioning, Neurobiol Learn Mem 94 (2010) 353-363.

[75] R.A. Rescorla, Effect of a stimulus intervening between CS and US in autoshaping, J Exp Psychol Anim Behav Process 8 (1982) 131-141.

[76] E.P. Riley, C.L. McGee, Fetal alcohol spectrum disorders: an overview with emphasis on changes in brain and behavior, Exp Biol Med 230 (2005) 357-365.

[77] R.C. Rizley, R.A. Rescorla, Associations in second-order conditioning and sensory preconditioning, J Comp Physiol Psychol 81 (1972) 1-11.
[78] S.H. Ryan, J.K. Williams, J.D. Thomas, Choline supplementation attenuates learning deficits associated with neonatal alcohol exposure in the rat: effects of varying the timing of choline administration, Brain Res 1237 (2008) 91-100.

[79] P.D. Sampson, B. Kerr, H.C. Olson, A.P. Streissguth, E. Hunt, H.M. Barr, et al., The effects of prenatal alcohol exposure on adolescent cognitive processing: a speedaccuracy tradeoff, Intelligence 24 (1997) 329-353.

[80] P.D. Sampson, A.P. Streissguth, F.L. Bookstein, R.E. Little, S.K. Clarren, P. Dehaene, et al., Incidence of fetal alcohol syndrome and prevalence of alcohol-related neurodevelopmental disorder, Teratology 56 (1997) 317-326.

[81] W.B. Schreiber, S.A. St. Cyr, S.A. Jablonski, P.S. Hunt, A.Y. Klintsova, M.E. Stanton, Effects of exercise and environmental complexity on deficits in trace and contextual fear conditioning produced by neonatal alcohol exposure in rats, Dev Psychobiol 55 (2013) 483-495

[82] W.B. Schreiber, P.S. Hunt, Deficits in trace fear conditioning induced by neonatal alcohol exposure persist into adulthood, Dev Psychobiol 55 (2013) 352-360.

[83] K. Stratton, C. Howe, F. Battaglia (Eds.), Institute of Medicine. Fetal Alcohol Syndrome: Diagnosis, Epidemiology, Prevention, and Treatment, National Academy Press, Washington DC1996.

[84] A.P. Streissguth, F.L. Bookstein, H.M. Barr, S. Press, P.D. Sampson, A fetal alcohol behavior scale, Alcohol Clin Exp Res 22 (1998) 325-333.

[85] B.L. Summers, A.M. Rofe, P. Coyle, Dietary zinc supplementation throughout pregnancy protects against fetal dysmorphology and improves postnatal survival after prenatal ethanol exposure in mice, Alcohol Clin Exp Res 33 (2009) 591-600.

[86] R.J. Sutherland, R.J. McDonald, D.D. Savage, Prenatal exposure to moderate levels of ethanol can have long-lasting effects on hippocampal synaptic plasticity in adult offspring, Hippocampus 7 (1997) 232-238.

[87] K. Takehara, S. Kawahara, Y. Kirino, Time-dependent reoraganization of the brain components underlying memory retention in trace eyeblink conditioning, J Neurosci 23 (2003) 9897-9905.

[88] J.D. Thomas, J.S. Biane, K.A. O'Bryan, T.M. O'Neill, H.D. Dominguez, Choline supplementation following third-trimester-equivalent alcohol exposure attenuates behavioral alterations in rats, Behav Neurosci 121 (2007) 120-130.

[89] J.D. Thomas, M.H. La Fiette, V.R.E. Quinn, E.P. Riley, Neonatal choline supplementation ameliorates the effects of prenatal alcohol exposure on a discrimination learning task in rats, Neurotoxicol Teratol 22 (2000) 703-711.

[90] J.D. Thomas, T.M. O'Neill, H.D. Dominguez, Perinatal choline supplementation does not mitigate motor coordination deficits associated with neonatal alcohol exposure in rats, Neurotoxicol Teratol 26 (2004) 223-229.

[91] J.D. Thomas, T.M. Sather, L.A. Whinery, Voluntary exercise influences behavioral development in rats exposed to alcohol during the neonatal brain growth spurt, Behav Neurosci 122 (2008) 1264-1273.

[92] J.D. Thomas, T.D. Tran, Choline supplementation mitigates trace, but not delay, eyeblink conditioning deficits in rats exposed to alcohol during development, Hippocampus 22 (2012) 619-630.

[93] V. Tiwari, K. Chopra, Resveratrol prevents alcohol-induced cognitive deficits and brain damage by blocking inflammatory signaling and cell death cascade in neonatal rat brain, J Neurochem 117 (2011) 678-690.

[94] T.D. Tran, S.J. Kelly, Critical periods for ethanol-induced cell loss in the hippocampa formation, Neurotoxicol Teratol 25 (2003) 519-528.

[95] T.D. Tran, M.E. Stanton, C.R. Goodlett, Binge-like ethanol exposure during the early postnatal period impairs eyeblink conditioning at short and long CS-US intervals in rats, Dev Psychobiol 49 (2007) 589-605.

[96] A. Uecker, L. Nadel, Spatial but not object memory impairments in children with fetal alcohol syndrome, Am J Ment Retard 103 (1998) 12-18.

[97] A.F. Wagner, P.S. Hunt, Impaired trace fear conditioning following neonatal ethanol: reversal by choline, Behav Neurosci 120 (2006) 482-487.

[98] K.A. Willoughby, E.D. Sheard, K. Nash, J. Rovet, Effects of prenatal alcohol exposure on hippocampal volume, verbal learning, and verbal and spatial recall in late childhood, J Int Neuropsychol Soc 14 (2008) 1022-1033.

[99] S.H. Zeisel, The fetal origins of memory: the role of dietary choline in optimal brain development, J Pediatr 149 (2006) S131-S136. 